PROCEEDINGS OF THE

AMERICAN MATHEMATICAL SOCIETY

Volume 124, Number 12, December 1996, Pages 3905-3911

S 0002-9939(96)03563-0

\title{
AN INEQUALITY FOR POLYHEDRA AND IDEAL TRIANGULATIONS OF CUSPED HYPERBOLIC 3-MANIFOLDS
}

\author{
MASAAKI WADA, YASUSHI YAMASHITA, AND HAN YOSHIDA \\ (Communicated by Ronald Stern)
}

\begin{abstract}
It is not known whether every noncompact hyperbolic 3-manifold of finite volume admits a decomposition into ideal tetrahedra. We give a partial solution to this problem: Let $M$ be a hyperbolic 3-manifold obtained by identifying the faces of $n$ convex ideal polyhedra $P_{1}, \ldots, P_{n}$. If the faces of $P_{1}, \ldots, P_{n-1}$ are glued to $P_{n}$, then $M$ can be decomposed into ideal tetrahedra by subdividing the $P_{i}$ 's.
\end{abstract}

\section{INTRODUCTION}

The famous lecture notes on 3-manifolds by Thurston [3] start with a decomposition of the figure eight knot complement into two ideal hyperbolic tetrahedra. Such a decomposition gives a convenient standpoint for the study of hyperbolic 3-manifolds, such as calculating deformations of the hyperbolic structure and computation of geometrical invariants [2]. It is thus natural to ask:

Question. Let $M$ be a noncompact hyperbolic 3-manifold of finite volume. Can we decompose $M$ into ideal tetrahedra?

Although Epstein and Penner have shown that such a manifold always admits a decomposition into convex ideal polyhedra [1], the answer to this question is not yet known. In [4], one of the authors has given an affirmative answer to the above question in the case where $M$ is obtained by gluing two convex ideal polyhedra in a certain way. In this paper, we generalize this result to the case where $M$ is composed of any number of convex ideal polyhedra:

Main Theorem. Let $M$ be a non-compact complete hyperbolic 3-manifold of finite volume which is obtained from $n$ convex ideal polyhedra $P_{1}, \ldots, P_{n}$ by identifying the faces in pairs. Suppose that each face of $P_{i}(i=1, \ldots, n-1)$ is pasted with a face of $P_{n}$, and the possibly remaining faces of $P_{n}$ glued in pairs. Then $M$ can be decomposed into non-degenerate ideal tetrahedra by subdividing the $P_{i}$ 's.

The key to our result is an inequality for polyhedra. Besides Euler's theorem and a few obvious equalities, not much seems to be known about the relations among the numbers of vertices, edges, and faces of a polyhedron; we feel that our inequality

Received by the editors June 15, 1995.

1991 Mathematics Subject Classification. Primary 57Q15, 52B05; Secondary 57M50, 51M20.

Key words and phrases. Hyperbolic 3-manifold, triangulation.

(C)1996 American Mathematical Society 

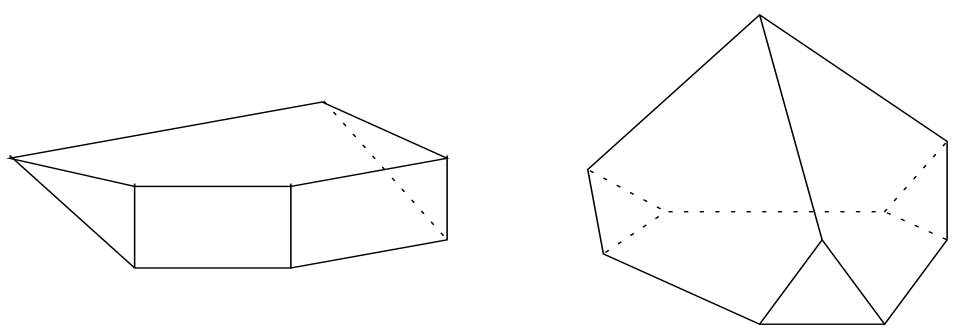

Figure 1

is of interest in its own right. We first introduce this inequality, and then prove the Main Theorem in section 3.

\section{AN INEQUALITY FOR POLYHEDRA}

Let $P$ be a polyhedron, and $V=V(P), E=E(P), F=F(P)$, and $F_{d}=F_{d}(P)$ denote respectively the sets of vertices, edges, faces, and $d$-gonal faces of $P$.

Theorem 1. In the notation above, we have

$$
|V|(|V|-1) \geq 8\left|F_{4}\right|+\sum_{d \geq 5} d(d-1)\left|F_{d}\right| .
$$

Equality in the above holds if and only if $P$ is combinatorially equivalent to one of the polyhedra depicted in Figure 1.

There is a slightly different way to view the inequality. A line segment connecting two vertices of $P$ is called a diagonal of $P$ if it goes through the interior of $P$; the set of diagonals of $P$ is denoted by $\Delta(P)$. Among the $\frac{1}{2}|V|(|V|-1)$ line segments connecting two vertices of $P$, the number of those which lie entirely in the boundary of $P$ is $\sum_{d \geq 3} \frac{1}{2} d(d-2)\left|F_{d}\right|$. Therefore Theorem 1 may be restated as:

Theorem 2. The number of diagonals of a polyhedron $P$ satisfies

$$
|\Delta(P)| \geq-\frac{3}{2}\left|F_{3}(P)\right|+\sum_{d \geq 5} \frac{d}{2}\left|F_{d}(P)\right| .
$$

Equality in the above holds if and only if $P$ is combinatorially equivalent to one of the polyhedra shown in Figure 1.

For our purposes, it is convenient to introduce the following notion.

Definition. For a polygon $f$ we define $D(f)$ by

$$
D(f)= \begin{cases}0 & \text { if } \operatorname{deg} f=3 \\ 2 & \text { if } \operatorname{deg} f=4 \\ d-1 & \text { if } \operatorname{deg} f=d \geq 5\end{cases}
$$

The capacity of $f$ is defined to be

$$
C(f)=D(f) \operatorname{deg} f
$$

It depends only on the degree of $f$; we also write $C(d)$ for the capacity of a $d$-gon. Finally the capacity of a sequence $\left(d_{1}, \ldots, d_{r}\right)$ of integers with $d_{i} \geq 3$ is defined to be the sum $\sum_{i=1}^{r} C\left(d_{i}\right)$. 
In terms of capacity, the inequality (1) is written as

$$
|V|(|V|-1) \geq \sum_{f \in F} C(f) .
$$

Proof of Theorem 1. Suppose that $|V|=p,|E|=q,|F|=r$, and that the faces of $P$ are of degree $d_{1}, \ldots, d_{r}$. We may assume that

$$
r-1 \geq d_{1} \geq \cdots \geq d_{r} \geq 3 .
$$

Since $q=\frac{1}{2} \sum_{i=1}^{r} d_{i}$, Euler's theorem implies that

$$
p=2+\frac{1}{2} \sum_{i=1}^{r} d_{i}-r
$$

We divide the proof of Theorem 1 into two cases. First suppose that $r \geq 9$. In this case the inequality (2) is a direct consequence of the following lemma:

Lemma. Let $\left(d_{1}, \ldots, d_{r}\right)$ be a sequence of integers satisfying (3). If $r \geq 9$ then for $p$ defined by (4) we have

$$
p(p-1)>\sum_{i=1}^{r} C\left(d_{i}\right)
$$

Note that the existence of a polyhedron whose faces are of degree $d_{1}, \ldots, d_{r}$ is not assumed.

Proof. Fix $r \geq 9$ and $p$. Of the sequences $\left(d_{1}, \ldots, d_{r}\right)$ satisfying the conditions (3) and $(4)$, choose one that attains the maximal capacity and denote it by $\left(d_{1}^{\prime}, \ldots, d_{r}^{\prime}\right)$. Clearly, it suffices to show the inequality (5) for these "maximal sequences."

Claim. There is $k$ such that

$$
r-1=d_{1}^{\prime}=\cdots=d_{k-1}^{\prime} \geq d_{k}^{\prime}>d_{k+1}^{\prime}=\cdots=d_{r}^{\prime}=3 .
$$

We prove the claim by contradiction; assume that $r-1>d_{i}^{\prime} \geq d_{j}^{\prime}>3$ for some $i$ and $j$. Consider replacing $\left(d_{i}^{\prime}, d_{j}^{\prime}\right)$ by $\left(d_{i}^{\prime}+1, d_{j}^{\prime}-1\right)$. The resulting sequence would

\begin{tabular}{|c|c|c|c|c|c|c|c|}
\hline$d$ & 3 & 4 & 5 & 6 & 7 & 8 & 9 \\
\hline$C(d)$ & 0 & 8 & 20 & 30 & 42 & 56 & 72 \\
\hline$C(d+1)-C(d)$ & & & & & & & \\
\hline
\end{tabular}
still satisfy the conditions (3) and (4), and the maximality of $\sum_{i=1}^{r} C\left(d_{i}^{\prime}\right)$ implies

$$
C\left(d_{i}^{\prime}\right)+C\left(d_{j}^{\prime}\right) \geq C\left(d_{i}^{\prime}+1\right)+C\left(d_{j}^{\prime}-1\right) .
$$

As we can see from the table below, this can occur only if

$$
\left(d_{i}^{\prime}, d_{j}^{\prime}\right)=(5,5) \text { or }(6,5)
$$

However, since

$$
\begin{aligned}
& C(5)+C(5)=40<42=C(7)+C(3), \\
& C(6)+C(5)=50<56=C(8)+C(3),
\end{aligned}
$$

replacing the pair $(5,5)$ or $(6,5)$ respectively by $(7,3)$ or $(8,3)$ would produce a sequence of greater capacity than $\left(d_{1}^{\prime}, \ldots, d_{r}^{\prime}\right)$; the replacement is allowed for $r \geq 9$. This contradiction proves the claim. 
Now we go back to the proof of the lemma. We write

$$
\begin{aligned}
d_{1}^{\prime}=\cdots=d_{k-1}^{\prime} & =s+3, \\
d_{k}^{\prime} & =t+3,
\end{aligned}
$$

thus

$$
0 \leq t \leq s=r-4
$$

By the above claim, we can compute $p$ and the capacity of $\left(d_{1}^{\prime}, \ldots, d_{r}^{\prime}\right)$ as follows:

$$
\begin{aligned}
& p=2+\frac{1}{2} \sum_{i=1}^{r} d_{i}^{\prime}-r \\
&=2+\frac{1}{2}\{(k-1)(r-1)+(t+3)+3(r-k)\}-r \\
&=\frac{1}{2}(k s+t+8), \\
& \sum_{i=1}^{r} C\left(d_{i}^{\prime}\right)=(k-1)(s+3)(s+2)+(t+3)(t+2) .
\end{aligned}
$$

Hence we have

$$
\begin{aligned}
& 4\left(p(p-1)-\sum_{i=1}^{r} C\left(d_{i}^{\prime}\right)\right) \\
& =(k s+t+8)(k s+t+6)-4(k-1)(s+3)(s+2)-4(t+3)(t+2) \\
& =\left(k s+t-2 s-3-\frac{12}{s}\right)^{2}+4 t(s-t)+24 \frac{t}{s}+8 s-9-\frac{72}{s}-\frac{144}{s^{2}} .
\end{aligned}
$$

It is easy to verify that

$$
8 s-9-\frac{72}{s}-\frac{144}{s^{2}}>0
$$

when $s=r-4 \geq 5$. Therefore we have

$$
p(p-1)>\sum_{i=1}^{r} C\left(d_{i}^{\prime}\right) .
$$

The lemma has been proved.

Next consider the case where $r<9$. Running a computer program shows that there are twelve sequences $\left(d_{1}, \ldots, d_{r}\right)$ which satisfy $(3)$ and

$$
p(p-1) \leq \sum_{i=1}^{r} C\left(d_{i}\right) .
$$

Of the twelve sequences, only $(5,5,4,4,3,3)$ and $(6,5,5,5,3,3,3)$ satisfy the following two necessary conditions for polyhedra:

$$
p \geq d_{1}+d_{2}+d_{3}-6
$$

since each pair of faces share at most two vertices, and

$$
3 p \leq \sum_{i=1}^{r} d_{i}
$$


since every vertex is incident with at least three faces. For the two sequences given above, which correspond to the polyhedra shown in Figure 1, the equality in (2) holds. This completes the proof of Theorem 1 .

The following corollary of Theorem 1 will be needed in the proof of the Main Theorem.

Corollary 3. Let $P$ be a polyhedron. Then there exists a vertex $v \in V(P)$ such that

$$
\sum_{v \in f \in F(P)} D(f) \leq|V(P)|-2 .
$$

Proof. One can easily check the statement for the two polyhedra shown in Figure 1; we assume that $P$ is neither of the two polyhedra. If

$$
|V(P)|-1 \leq \sum_{v \in f} D(f)
$$

for all $v \in V(P)$, then we would have

$$
\begin{aligned}
|V(P)|(|V(P)|-1) & \leq \sum_{v \in V(P)} \sum_{v \in f} D(f) \\
& =\sum_{f \in F(P)} C(f) .
\end{aligned}
$$

However this contradicts Theorem 1. Therefore there must be a vertex $v \in V(P)$ satisfying (6).

\section{Proof of the Main Theorem}

Let $M$ be a 3-manifold obtained from convex ideal polyhedra $P_{1}, \ldots, P_{n}$ by identifying the faces in pairs. A pair of faces which are to be identified are called partner faces of each other. We denote by $F^{(i)}\left(P_{j}\right)$ the set of faces $f \in F\left(P_{j}\right)$ whose partner face belongs to $F\left(P_{i}\right)$.

We first introduce what we call a "cone decomposition" of a convex polyhedron. Let $P$ be a convex polyhedron, and $v \in V(P)$ a vertex of $P$. Divide those faces of $P$ not containing $v$ into triangles (in any way you like), and the polyhedron $P$ is decomposed into the cones of these triangles from the vertex $v$. This decomposition is called a cone decomposition of $P$ from $v$. Note that taking arbitrary cone decompositions of $P_{1}, \ldots, P_{n}$ does not in general result in a proper decomposition of $M$, for the induced triangulations of partner faces may not coincide when the faces are identified; although this difficulty can be easily avoided if we are allowed to insert degenerate (flattened) ideal tetrahedra between such incompatible pairs of partner faces.

We will show, under the assumption that each face of $P_{i}(i=1, \ldots, n-1)$ is identified with a face of $P_{n}$, that the above incompatibility does not occur if we carefully choose cone decompositions of $P_{1}, \ldots, P_{n}$. For $i=1, \ldots, n-1$, we choose a vertex $v_{i} \in V\left(P_{i}\right)$ given by Corollary 3 so that

$$
\sum_{v_{i} \in f \in F\left(P_{i}\right)} D(f) \leq\left|V\left(P_{i}\right)\right|-2
$$

and take a cone decomposition of $P_{i}$ from $v_{i}$. 
Now consider choosing a vertex $v_{n} \in V\left(P_{n}\right)$, and taking a cone decomposition of $P_{n}$ from $v_{n}$. Recall that, when taking a cone decomposition of a polyhedron $P$ from a vertex $v$, we can freely triangulate those faces of $P$ not containing $v$. Hence, by choosing a cone decomposition of $P_{i}$ and one of $P_{n}$ appropriately, we can make the induced triangulations of the faces of $P_{i}$ and of $P_{n}$ compatible; unless there are partner faces $f \in F\left(P_{i}\right)$ and $f^{\prime} \in F\left(P_{n}\right)$ such that $v_{i} \in f, v_{n} \in f^{\prime}$, and either

(1) $\operatorname{deg} f=\operatorname{deg} f^{\prime}=4$, and $v_{i}$ is adjacent to $v_{n}$ when $f$ and $f^{\prime}$ are identified, or

(2) $\operatorname{deg} f=\operatorname{deg} f^{\prime} \geq 5$, and $v_{i}$ does not correspond to $v_{n}$ under the identification.

Let $A_{i}$ denote the set of vertices $v_{n} \in V\left(P_{n}\right)$ from which every cone decomposition of $P_{n}$ causes an incompatibility problem of facial triangulations between $P_{i}$ and $P_{n}$. Then by the above we have

$$
\left|A_{i}\right| \leq \sum_{v_{i} \in f} D(f) \leq\left|V\left(P_{i}\right)\right|-2 .
$$

We also need to choose a cone decomposition of $P_{n}$ from $v_{n}$ so that the induced triangulations of each pair of partner faces of $P_{n}$ coincide under the identification. This can be done unless there exist a pair of partner faces $f, f^{\prime} \in F^{(n)}\left(P_{n}\right)$ sharing the vertex $v_{n}$ such that either

(1) $\operatorname{deg} f=\operatorname{deg} f^{\prime}=4$, and the gluing map sends $v_{n}$ to one of the vertices of $f^{\prime}$ adjacent to $v_{n}$, or

(2) $\operatorname{deg} f=\operatorname{deg} f^{\prime} \geq 5$, and the identification does not map $v_{n}$ to itself.

Let $A_{n}$ denote the set of vertices $v_{n} \in V\left(P_{n}\right)$ from which every cone decomposition of $P_{n}$ induces incompatible triangulations on some pair of partner faces of $P_{n}$. Since two faces can share at most two vertices, we have

$$
\left|A_{n}\right| \leq \sum_{d \geq 4}\left|F_{d}^{(n)}\left(P_{n}\right)\right|
$$

where $F_{d}^{(n)}\left(P_{n}\right)$ denotes the set of faces $f \in F^{(n)}\left(P_{n}\right)$ of degree $d$.

To prove the theorem, it suffices to show that there exists a vertex of $P_{n}$ not contained in any of the sets $A_{1}, \ldots, A_{n}$. We show this by proving $\left|V\left(P_{n}\right)\right|>\sum_{i=1}^{n}\left|A_{i}\right|$ as follows:

$$
\begin{aligned}
\left|V\left(P_{n}\right)\right|-2 & =\left|E\left(P_{n}\right)\right|-\left|F\left(P_{n}\right)\right| \\
& =\sum_{f \in F\left(P_{n}\right)}\left(\frac{1}{2} \operatorname{deg} f-1\right) \\
& =\sum_{i=1}^{n} \sum_{f \in F^{(i)}\left(P_{n}\right)}\left(\frac{1}{2} \operatorname{deg} f-1\right) .
\end{aligned}
$$

Here, for $i=1, \ldots, n-1$, we have

$$
\begin{aligned}
\sum_{f \in F^{(i)}\left(P_{n}\right)}\left(\frac{1}{2} \operatorname{deg} f-1\right) & =\sum_{f \in F\left(P_{i}\right)}\left(\frac{1}{2} \operatorname{deg} f-1\right) \\
& =\left|V\left(P_{i}\right)\right|-2 \\
& \geq\left|A_{i}\right|
\end{aligned}
$$


and

$$
\begin{aligned}
\sum_{f \in F^{(n)}\left(P_{n}\right)}\left(\frac{1}{2} \operatorname{deg} f-1\right) & =\sum_{d \geq 3}\left(\frac{d}{2}-1\right)\left|F_{d}^{(n)}\left(P_{n}\right)\right| \\
& \geq \sum_{d \geq 4}\left|F_{d}^{(n)}\left(P_{n}\right)\right| \\
& \geq\left|A_{n}\right| .
\end{aligned}
$$

Therefore we have

$$
\left|V\left(P_{n}\right)\right|-2 \geq \sum_{i=1}^{n}\left|A_{i}\right| .
$$

This completes the proof of the Main Theorem.

\section{REFERENCES}

1. D. B. A. Epstein and R. Penner, Euclidean decomposition of noncompact hyperbolic manifolds, J.Differential Geom. 27 (1988), 67-80. MR 89a:57020

2. M. Hildebrand and J. Weeks, A comuputer generated census of cusped hyperbolic 3-manifolds, Computers and Mathematics (E. Kaltofen and S. Watt, eds.), Springer, Berlin, 1989, pp. 5359. MR 90f:57043

3. W. Thurston, The Geometry and Topology of Three-Manifolds, Lecture Notes, Princeton University, 1978.

4. H. Yoshida, Ideal tetrahedral decompositions of hyperbolic 3-manifolds, Osaka J. Math. 33 (1996), 37-46. CMP 96:10

Faculty of Science, Nara Women's University, Kita-Uoya Nishimachi, Nara 630, Japan

E-mail address: wada@ics.nara-wu.ac.jp

E-mail address: yamasita@ics.nara-wu.ac.jp

E-mail address: han@ics.nara-wu.ac.jp 